\title{
THE ULTRASTRUCTURE OF GERMINATING BARLEY SEEDS. II. BREAKDOWN OF STARCH GRANULES AND CELL WALLS OF THE ENDOSPERM IN THREE BARLEY VARIETIES
}

by

\author{
NIELS H. GRAM \\ Department of Physiology, Carlsberg Laboratory, \\ Gamle Carlsberg Vej 10. DK-2500 Copenhagen Valby \\ and \\ Department of Crop Husbandry and Plant Breeding \\ Royal Veterinary and Agricultural University \\ Thorvaldsensvej 40, DK-187I Copenhagen V
}

Keywords: Nordal, Minerva, Klages, freeze-fracturing, thin sectioning, corrosion channels

The breakdown during malting of starch granules and cell walls of the endosperm, as well as the morphological changes occurring in the scutellum and the aleurone layer, have been examined by thin sectioning and freeze-fracturing in the barley varieties Nordal, Minerva and Klages.

Intact starchy endosperm cells contain large and small starch granules embedded in a matrix. In most cases the fracture plane exposes the smooth surface of the starch granules and occasionally adhering material, which probably consists of remnants of the amyloplast envelope. Degradation of the endosperm tissue during maiting starts at the embryo end, and the transitional zone between intact and degraded endosperm is approximately one cell layer wide. The degradation of the large starch granules affects at the beginning only their outer edge. Subsequently, corroding channels form towards the center of the granules and elicit a rapid breakdown of the central part. Further degradation proceeds from the center giving rise to hollow shells, which finally break up into pieces. It is concluded that the susceptibility to enzyme attack increases towards the center of the large starch granules of the barley endosperm. The saw-toothed pattern observed in the corroding channels indicates, in agreement with earlier studies, that the enzyme susceptibility varies periodically in a radial direction.

The three barley varieties examined differed in malting quality, but the differences were not reflected qualitatively in the ultrastructure of the scutellum, aleurone layer or the endosperm during malting. 


\section{INTRODUCTION}

The starchy endosperm cells, containing starch granules and storage protein, constitute the major part of the mature barley seed. During development, protein is deposited in vacuoles (6), while the starch granules are formed within plastids $(4,5,22,23)$. Two distinct types of starch granules develop in the endosperm cells: Large and small granules $(4,5,15,18,20,22$, 23). The large granules develop up to 15 days after anthesis (15), and as the growth of the granules is more rapid in one plane, the large granules appear lense or bean-shaped. At this time the amyloplasts start to bud off stromafilled plastids, in which the small, spherical starch granules form $(4,5)$. The ratio of small to large granules differs from variety to variety (10), but in general the small granules constitute about $90 \%$ of the total number of granules, but only $10 \%$ of the total volume of starch (15). There is apparently no major difference between large and small granules with regard to the content of protein, lipid and amylopectin (11, 22). As the seed approaches maturity, the starch occupies most of the space in the endosperm cells, and at these late stages of development it is not possible to obtain ultrathin sections of satisfactory quality (4). Therefore little information is available about the ultrastructure of the endosperm cells at or near maturity. The starchy endosperm of mature seeds appears as a dead storage tissue, with only remnants of cell organelles present $(21)$.

During malting, the cell walls of the barley endosperm are degraded, starting in the embryo end of the seed and progressing towards the distal end as malting proceeds $(2,3,8,9)$. Furthermore, it has been reported that the small starch granules disappear first during malting, and the remaining large granules exhibit a pitted surface, indicating the initiation of their degradation $(17,19)$.

The present investigation was carried out to study the morphology of starch granules and cells walls of the endosperm during malting in three barley varieties by the use of freezefracturing and thin sectioning.

\section{MATERIALS AND METHODS}

\subsection{Micromalting}

The present analysis was performed on barley seeds, Hordeum vulgare L., varieties Nordal, Minerva and Klages, harvest 1981. The seeds, kindly provided by Dr. J. LARSEN, germinated in a micromalting device developed at the Carlsberg Laboratory. The device consists of a stainless steel box, which contains a rotating cylinder. The cylinder accomodates up to 144 samples of seeds, each sample weighing about 80 grams.

The steeping period lasted 28 hours, comprising 6 hours immersion in water and aeration with humidified air for 18 hours, followed by a second immersion of additional 4 hours. The steeping was carried out at $16^{\circ} \mathrm{C}$. Subsequently, the seeds germinated for 116 hours in humidified air with an aeration rate $71 / \mathrm{min}$. The temperature of the inlet air was $12-13^{\circ} \mathrm{C}$, and near the seeds, the temperature was $14-15^{\circ} \mathrm{C}$.

The duration of the entire program thus amounted to 144 hours. Samples for the freezefracture analysis were taken after 100 hours, and samples to be thin sectioned were taken after 0,72 and 144 hours.

\subsection{F reeze-fracturing}

In each sample, 10 seeds were randomly chosen. The seeds were stained with Calcofluor White M2R New as previously described (12) to analyse the extent of wall degradation in the endosperm. Small plugs $\left(1 \mathrm{~mm}^{3}\right)$ of tissue were then removed from the zone between modified and unmodified endosperm, and in the modified endosperm region near the embryo. The plugs were placed on gold specimen discs in a small drop of $20 \%$ glycerol and frozen as quickly as possible in melting Freon 22, cooled by liquid nitrogen.

Replicas were made with a Balzers BAF 301 freeze-etching device (Balzers $A G$, Liechtenstein). At a pressure of $5 \times 10^{-6}$ torr or less, the specimens were fractured with a single cut and etched for 2 minutes at a temperature of $-100^{\circ} \mathrm{C}$.

Following coating with platinum/carbon and carbon, the specimens were immersed in $100 \%$ glycerol and transferred to $80 \%$ sulphuric acid and held at $60^{\circ} \mathrm{C}$ for at least 3 hours (14) to release the replica from the endosperm tissue. 
The replicas were washed with distilled water and kept overnight in $15 \%$ hypochlorite. After 4 washes in distilled water, the replicas were picked up on 200 mesh copper grids. If replicas had sunk in the hypochlorite solution, they were transferred through distilled water, to acetone and back to distilled water.

The replicas were examined with a Siemens 102 electron microscope at $80 \mathrm{kV}$. All freezefracture micrographs are printed, so that the direction of shadowing is from the bottom to the top of the page.

\subsection{Fixation}

For thin sectioning, 10 seeds were randomly chosen in each sample. First, the seeds were cut in three parts by two longitudinal and parallel dorsoventral cuts. The two outer sections were not used in the analysis. The mid section, a 1.2 $\mathrm{mm}$ thick slice, was cleaved by another cut into two slices, each $0.6 \mathrm{~mm}$ thick. One of these slices was stained with Calcofluor and photographed as previously described (12). The average modification of the endosperm was estimated from the stained sections, and the five seeds, which showed a modification closest to the average, were chosen for the electron microscopic analysis.

The embryo part and the dorsal aleurone tissue with adhering endosperm tissue were cut out of the unstained slices, and the different parts of the seeds were fixed and embedded as previously described (12).

Ultrathin sections were analysed in a Siemens 102 electron microscope at $80 \mathrm{kV}$.

\section{RESULTS}

Intact cells of the starchy endosperm (Figures 1-4) consist of cell walls and large and small starch granules, embedded in a matrix. The cell walls, a few microns in thickness, appear after freeze-fracturing to have a granular substructure, with fibrils running in the longitudinal direction of the cell wall (Figure 2A). The middle lamella is seen as an electron dense band between the walls of neighbouring cells (Figures $\mathrm{IC}$ and 2A). In freeze-fracture replicas, the ground substance of the cells appears more or less granular (Figures 2B and 3A), while in ultrathin sections the matrix appears uniformly electron dense, occupying the space between the starch granules (Figure 1C). In unmodified endosperm cells the fracture plane passes mostly around the starch granules, revealing a smooth surface with local indentations (Figures 2B, 3A and 3B). The indentations are probably the result of the limited space during the later stages of starch deposition in the ciosely packed endosperm cells. In the few cases where the starch granules have been cleaved by the fracture plane (Figure 4), the content of the granules appears as a homogeneous, granular substance. BUTTROSE (4) reported that the large starch granules of barley endosperm cells have the shape of oblate spheroids with an equatorial groove extending around the granules. When cross-fractured starch granules in the present study revealed an oblate outline, a groove was often observed at the equatorial plane of the granule (denoted by an arrow in Figure 4B). It has been reported that the starch granules of mature wheat endosperm possess remnants of the plastid membrane (1). Material adhering to the surface of the starch granules was occasion. ally observed in the present study (denoted by arrows in Figure 2A), in agreement with the findings of BARLow et al. (1). However, in cases where the fracture plane revealed both the surface and the inside of the starch granules (Figure 4A), an amyloplast envelope at the periphery of the granule could not be identified. Likewise, ultrathin sectioning failed to demonstrate a double membrane envelope or remnants thereof (Figure 1C). No qualitative differences were observed in the ultrastructural morphology of the three barley varieties, the only difference being that the large starch granules of the variety Klages occasionally exhibited a pitted surface (Figure 3B), in contrast to the starch granules of Nordal and Minerva barley, which always appeared with a smooth surface.

The transitional zone between modified and unmodified endosperm in germinating barley seeds is narrow, as observed in freeze-fracture replicas. Within a distance of a few hundred microns, the cell walls of the endosperm and the small starch granules disappear. The present freeze-fracture analysis failed to reveal partially degraded small starch granules or cell walls. In thin sections cut near the aleurone layer, the 
N. H. Gram: The ultrastructure of germinating barley seeds. II.

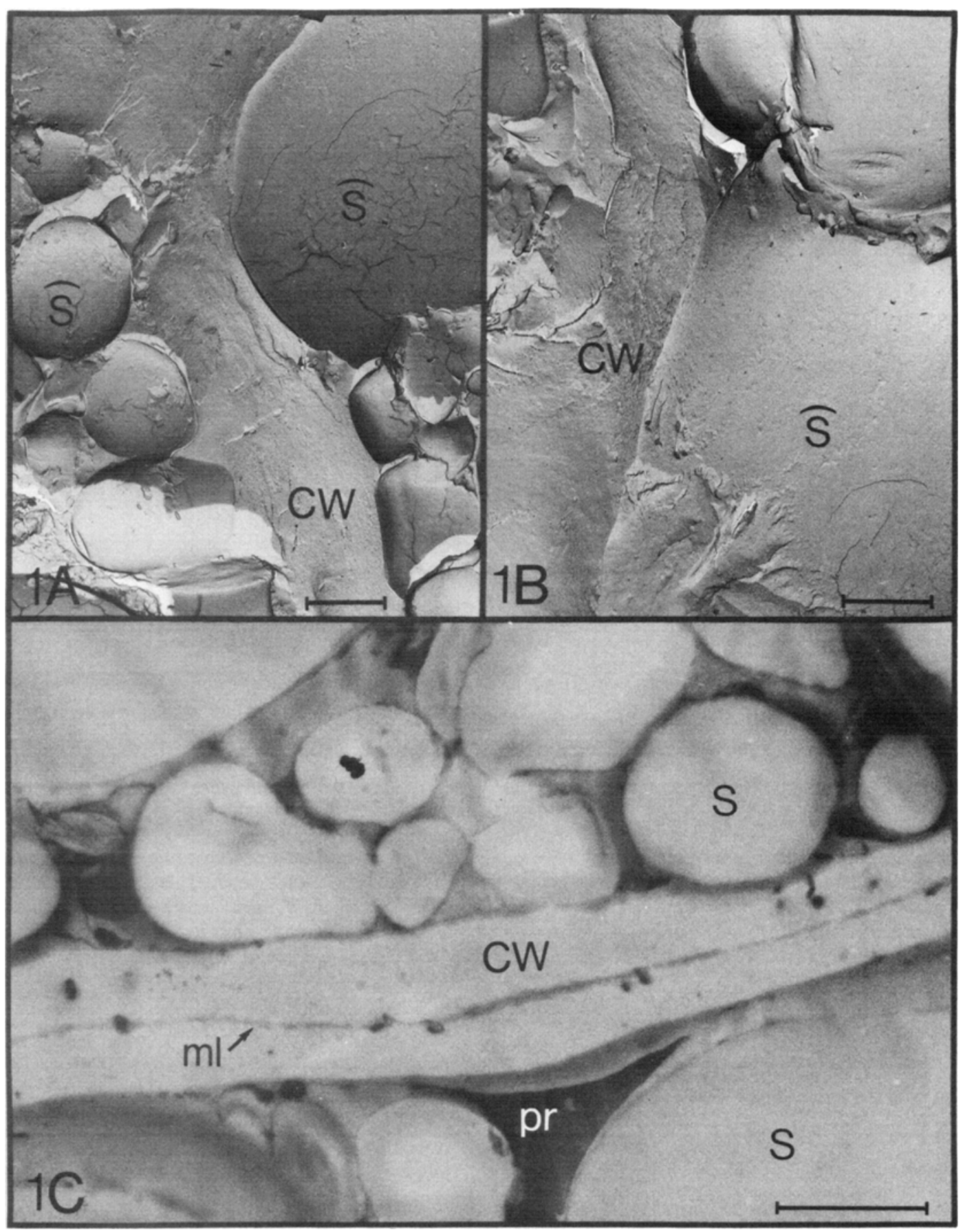

Figure 1. Freeze-fracture and thin section micrographs from intact starchy endosperm cells of Nordal barley. The freeze-fracture micrographs (Figures IA and IB) show cell walls (cw) and convex fractured starch granules $(\widehat{\mathrm{s}})$, appearing with a smooth surface. In the thin section micrograph (Figure IC) starch granules (s) and the cell wall (cw) with the middle lamella $(\mathrm{ml})$ are seen, together with the electron dense protein matrix (pr). The starch granules are apparently not surrounded by membranes. $($ Bar $=2 \mu \mathrm{m})$. 


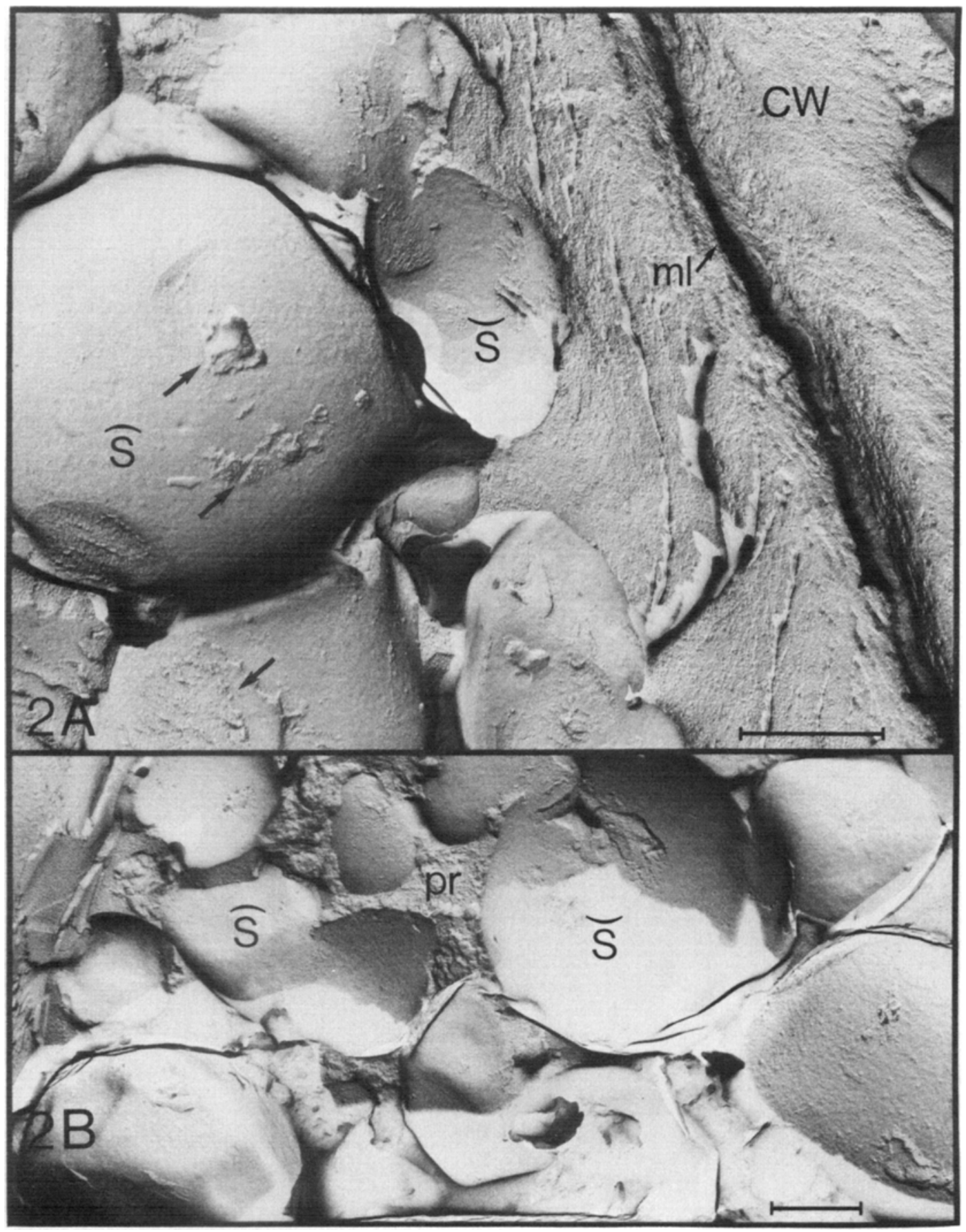

Figure 2. Freeze-fracture micrographs from intact starchy endosperm cells of Minerva barley.

Both convex $(\widehat{\mathrm{s}})$ and concave $(\widetilde{\mathrm{s}})$ fractured starch granules are seen. The cell wall (cw) in Figure 2A shows a fibrillar and granular substructure, and the middle lamella $(\mathrm{ml})$ is present as an electron dense groove between the walls. Note the material adhering to the granules in Figure 2A (denoted by arrows). The protein matrix (pr) appears granular in Figure 2B. A starch granule with indentations is present in the lower left corner of Figure 2B. $($ Bar $=1 \mu \mathrm{m})$. 


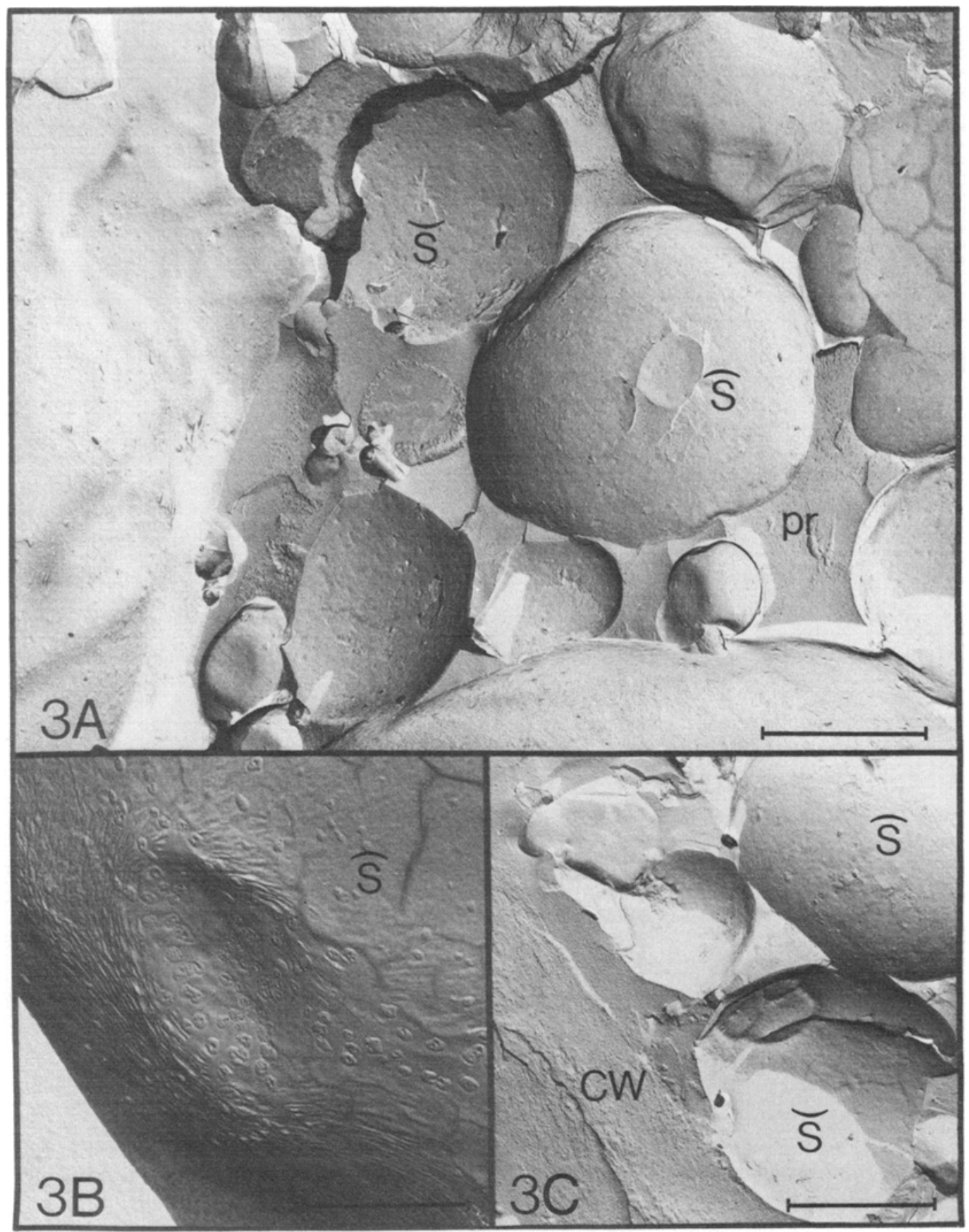

Figure 3. Freeze-fracture micrographs from intact starchy endosperm cells of Klages barley.

Smooth convex $(\widehat{\mathrm{s}})$ and concave $(\widetilde{\mathrm{s}})$ fractured starch granules are present. The protein matrix (pr) in Figure 3A appears to have a fine granular structure. At the top of Figure 3A, a granule with indentations can be seen. The large starch granules of Klages occasionally exhibit a pitted surface, as seen in Figure 3B. cw, cell wall. (Bar $=2 \mu \mathrm{m})$. 
N. H. Gram: The ultrastructure of germinating barley seeds. II.

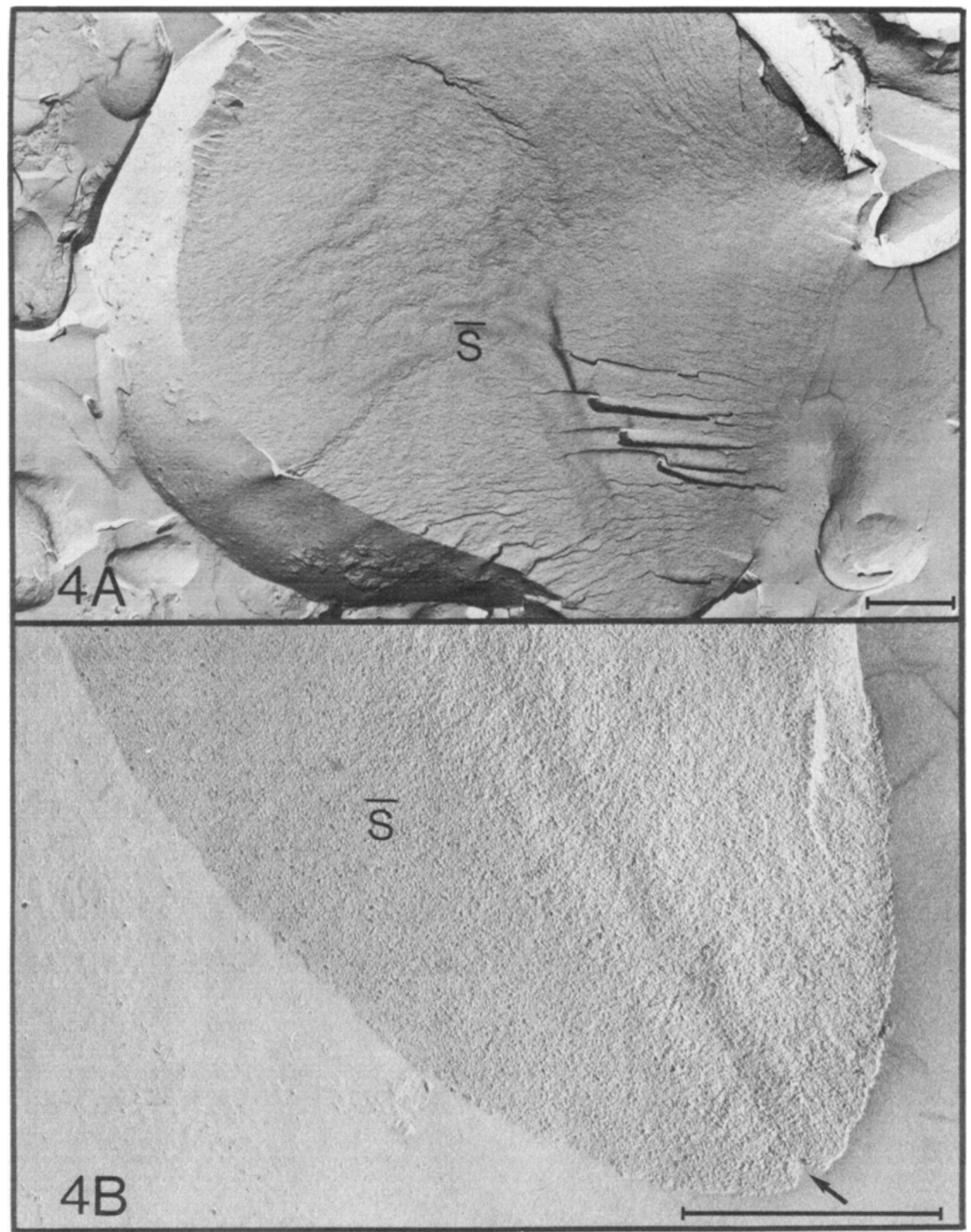

Figure 4. Cross-fractured starch granules $(\bar{s})$ from intact starchy endosperm cells.

The content of the granule in Figure 4A, from Minerva barley, appears as a homogeneous, granular substance. The granule is also partly surface-fractured, and it is not possible to detect a plastid membrane at the periphery of the granule. The granule in Figure $4 \mathrm{~B}$, from Nordal barley, reveals a groove at the equatorial plane (denoted by an arrow), which may encircle the granule. (Bar $=2 \mu \mathrm{m}$ ). 


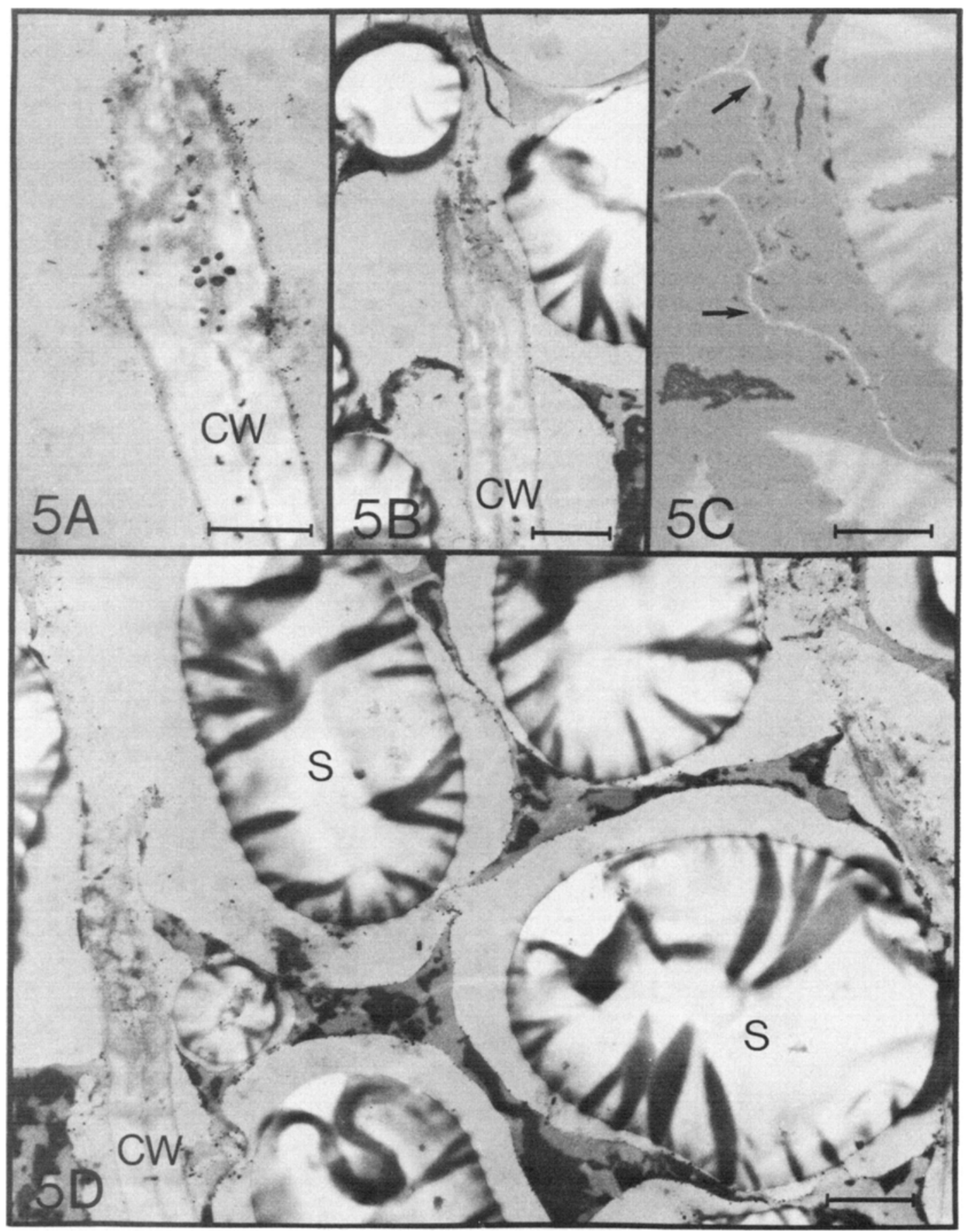

Figure 5. Micrographs illustrating the breakdown of the starchy endosperm cell walls in Nordal barley.

Figures 5A, 5B and 5D are micrographs from thin sections cut near the aleurone layer in seeds malted for 144 hours. The degradation is abrupt; apparently intact cell walls (cw) are seen at the bottom of Figures 5A, 5B and $5 \mathrm{D}$, whereas practically no wall material is detectable at the top of the same micrographs. Figure $5 \mathrm{C}$ shows a thin section cut near the scutellum in a seed malted for 72 hours. Note the electron transparent profiles (denoted by arrows), which are interpreted as remnants of the cell wall. $s$, starch granule. (Bar $=2 \mu \mathrm{m}$ ). 


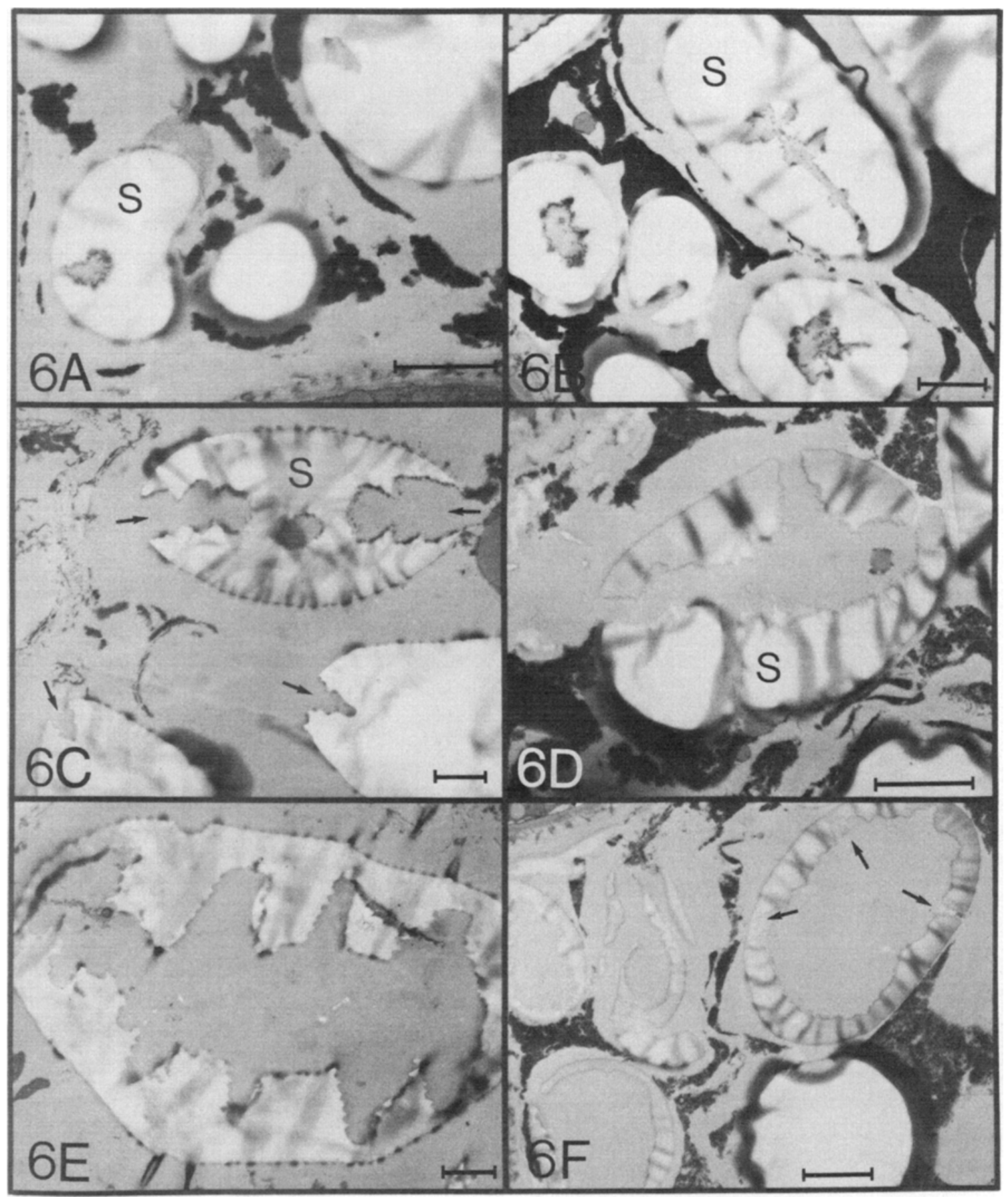

Figure 6. Thin section micrographs showing degrading starch granules located near the aleurone layer in Nordal (Figures 6A-6D and 6F) and Klages barley (Figure 6E).

Degradation appears to be initiated in limited regions at the periphery of the granules (Figure 6A), followed by the formation of corroding channels towards the center of the granules (Figures 6B, 6C and 6D). Subsequently, the center is degraded (Figure 6E), and almost completely degraded granules appear as starch shells with openings traversing the shell (denoted by arrows in Figure $6 \mathrm{~F}$ ). Note the saw-toothed pattern in the corroding channels of Figure $6 \mathrm{E}$, and that the corrosion often starts at the ends of the oblong starch granules (denoted by arrows in Figure 6C). (Bar $=2 \mu \mathrm{m})$. 
N. H. GRAM: The ultrastructure of germinating barley seeds. II.

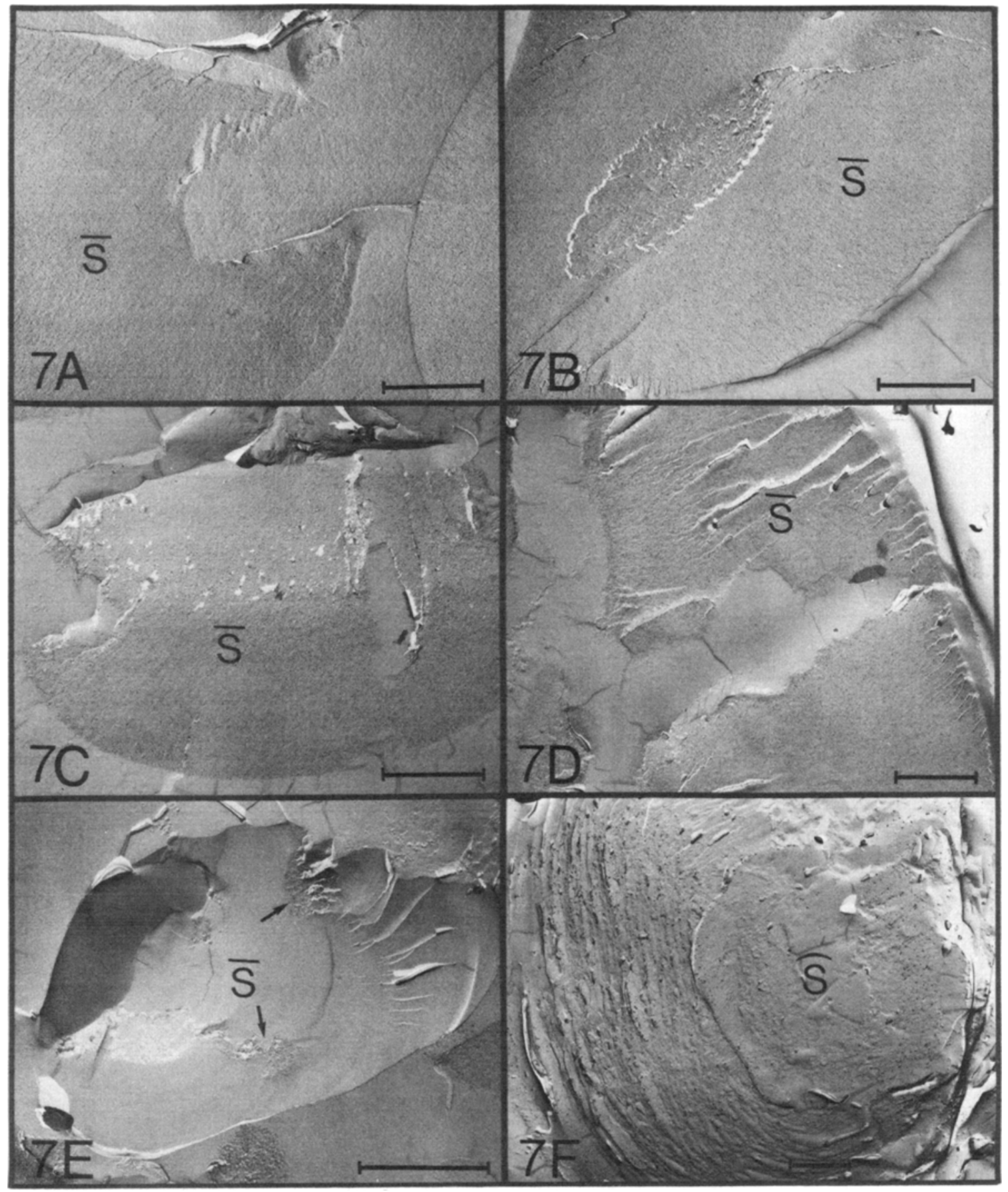

Figure 7. Freeze-fracture micrographs showing degrading starch granules located near the scutellum in Nordal barley seeds malted for 100 hours.

Corrosion channels are present in Figure $7 \mathrm{~A}, 7 \mathrm{C}$ and $7 \mathrm{D}$, while the corrosion in Figure $7 \mathrm{E}$ appears as restricted, granular areas (denoted by arrows). The fracture-face shown in Figure 7B reveals the central cavity of a partially degraded granule. The surface-fractured starch granule $(\widehat{\mathbf{s}})$ in Figure $7 \mathrm{~F}$ exhibits a lamellar structure. $\overline{\mathrm{s}}$, cross fractured starch granule. (Bar $=2 \mu \mathrm{m})$. 
breakdown of the cell walls could be seen to occur abruptly over a distance covering only a few microns (Figures 5A, 5B and 5D). In sections cut near the scutellum, thin electron transparent profiles are observed, which may represent cross sections through remnants of the middle lamella of the cell walls (Figure 5C).

In seeds malted for 100 hours or more, degradation of the large starch granules can be observed in thin sections cut near the aleurone layer or the scutellum (Figure 6), as well as in freeze-fracture replicas from well-modified endosperm tissue near the scutellum (Figure 7). The degradation apparently starts in restricted, small regions on the surface of the starch granule (Figure 6A), followed by the formation of corrosion channels towards the center of the granule (Figures 6B, 6C, 6D, 7A, 7C and 7D). The central part of the starch granule is degraded more rapidly than the rest of the granule (Figures $6 \mathrm{D}, 6 \mathrm{E}$ and $7 \mathrm{~B}$ ), and therefore almost completely degraded starch granules are shells, which appear in thin sections as ring-shaped structures with one or several openings in the ring (denoted by arrows in Figure 6F). A saw-toothed pattern is often observed in the corroding channels, indicating that the susceptibility to enzyme degradation varies periodically from the surface to the center of the granules. Furthermore, the degradation of the starch starts preferentially at the ends of the oblong granules, $i$. e. within the equatorial groove (Figure 6C). Freeze-fracture replicas from well-modified endosperm tissue reveal mainly cross-fractured starch granules. In those cases where the granules have been surface-fractured, a lamellar structure is often observed (Figure 7F).

Together with the examination of the starchy endosperm, the ultrastructural changes occurring in the scutellum and the aleurone layer were examined. The ultrastructural changes in $\mathrm{Mi}$ nerva and Klages were identical to those previously described for the variety Nordal (12). The three barley varieties differed in their malting quality: Nordal is a standard malting variety, Minerva yields malt extracts with increased viscosity, and Klages malts more rapidly. The differences in malting quality were not reflected in qualitative differences in the ultrastructure of the starchy endosperm, the scutellum or the aleurone layer during malting.

\section{DISCUSSION}

The starch granules of the developing barley endosperm are formed inside the double membrane bound amyloplasts (4). The results of the present investigation show that intact plastid envelopes are no longer present around the starch granules in the mature, germinating seed. In freeze-fracture replicas, only pieces of adhering material, which may be remnants of the amyloplast envelope are observed on the surface of the starch granules (Figure 2A), and in thin sections membranes surrounding the granules cannot be identified (Figure IC).

Germinating seeds contain both intact and degraded starchy endosperm tissue, separated by a narrow transitional zone, about one cell layer in thickness. Endosperm cells adjacent to degraded endosperm tissue appear identical to endosperm cells of ungerminated seeds, i.e. having intact cell walls, large and small starch granules and storage protein. This shows that the cell walls of the endosperm constitute an effective barrier to the diffusion of the enzymes hydrolysing the starch and protein of the germinating seed. Apparently, the cell walls must be degraded by the glucanases before the amylases and proteinases can reach the cell content and degrade it.

Several reports have shown that the degradation of the starch granules in germinating seeds from leguminous species starts from the interior of the granules, and that mitochondria and endoplasmic reticulum invade the cavity formed in the granules $(7,13,16)$. In the present study, cell organelles were not observed in the starchy endosperm tissue at any stage of malting. The corrosion pattern of the large starch granules demonstrates that the susceptibility to enzyme attack increases towards the center of the large granules, which is also supported by the fact that the corroding channels enlarge towards the center (Figures 6D, 6E and 7D). The equatorial plane of the large starch granules obviously represents the more susceptible part of the granule surface, since the formation of corrosion channels most readily occurs along this plane (Figure 6C). Furthermore, the walls of the corroding channels often exhibit a characteristic saw-toothed pattern, indicating alternating layers of higher and lower resistance to amylase degradation in a radial direction (Figure 6E). 
N. H. Gram: The ultrastructure of germinating barley seeds. II

This saw-toothed pattern corresponds to the well-known concentric layer structure of starch granules observed in the light microscope (20) and in the electron microscope after potassium permanganate fixation and staining, as well after hydrochloric acid treatment (4). As BuTTROSE (4) has shown, each itooth " reflects a layer deposited during a single day's growth of the starch granule, the composition of the starch deposited during the day being different from that made in the evening and the morning. Under constant light and temperature no lamination of the starch granules are observed and no saw-toothed pattern.

\section{ACKNOWLEDGEMENTS}

I wish to express my sincere gratitude to Professor Diter voN WeTtSTEIN, who besides suggesting the investigation also greatly influenced this work through inspiring guidance and discussions together with Dr. Søren Rasmussen and Dr. Preben Holm. Their assistance in preparing this communication is also highly appreciated. I especially thank BIBI STAMPE ANDERSEN for outstanding technical assistance, Nina RASMUSSEN for preparing the figures, LeNa KONGSRUD for typing the manuscript and Dr. JøRGEN LARSEN for providing the seeds. Finally, the assistance of Bent HanSEN, ANN-Sofi SteInHOLTZ and JEAN SAGE is gratefully acknowledged.

\section{REFERENCES}

1. Barlow, K., M. S. Buttrose. D. H. Simmonds \& M. VESK: The nature of the starch-protein interface in wheat endosperm. Cereal Chem. 50 , 443-454 (1973)

2. BrigGs, D. E.: Enzyme formation, cellular breakdown and the distribution of gibberellins in the endosperm of barley. Planta 108, 351-358 (1972)

3. Brown, H. T. \& G. H. Morris: Researches on the germination of some of the Graminex. J. Chem. Soc. 57, 458-528 (1890)

4. Butrrose, M. S.: Submicroscopic development and structure of starch granules in cereal endosperms. J. Ultrastructure Res. 4, 231-257 (1960)

5. ButTrose, M. S.: Ultrastructure of the develop- ing wheat endosperm. Austr. J. Biol. Sci. 16. 305-317 (1963)

6. Cameron-Milus, V. \& D. von Wettstein: Protein body formation in the developing barley endosperm. Carlsberg Res. Commun. 45, 577594 (1980)

7. Chandorkar, K. R. \& N. P. Badenhuizen: The fate of ADPG-alpha-glucan glucosyltransferase during amylolytic corrosion of starch granules, and its relation to starch granule structure. Cereal Chem. 44, 27-38 (1967)

8. Gibbons, G. C.: On the sequential determination of $\alpha$-amylase transport and cell wall breakdown in germinating seeds of Hordeum vulgare. Carlsberg Res. Commun. 45, 177-184 (1980)

9. Gibbons, G. C.: On the relative role of the scutellum and aleurone in the production of hydrolases during germination of barley. Carlsberg Res. Commun. 46, 215-225 (1981)

10. Goering, K. J., D. H. Fritts \& R. F. Eslick: A study of starch granule size and distribution in 29 barley varieties. Stäke 25, 297-302 (1973)

11. Goering, K. J. \& B. DeHaAs: A comparison of the properties of large- and small-granule starch isolated from several isogenic lines of barley Cereal Chem. 51, 573-578 (1974)

12. GRAM, N. H.: The ultrastructure of germinating barley seeds. I. Changes in the scutellum and the aleurone layer in Norda! Barley. Carlsberg Res. Commun. 47, 143-162 (1982)

13. HaRRIS, N.: Starch grain breakdown in cotyledon cells of germinating Mung bean seeds. Planta 129. 271-272 (1976)

14. LotT, J. A. \& M. S. Butrrose: Thin sectioning, freeze fracturing, enzyme dispersive X-ray anal$y s i s$, and chemical analysis in the study of inclusions in seed protein bodies: almond. Brazil nut, and quandong. Can. J. Bot. 56, 2050-2061 (1978)

15. May, L. H. \& M. S. Butrrose: Physiology of cereal grain. II. Starch granule formation in the developing barley kernel. Austr. J. Biol. Sci. 12 , 146-159 (1959)

16. NaYYaR, V. L. \& H. Y. Mohan Ram: Ultrastructure of starch grain breakdown in cotyledons of Cajanus cajan during germination. Phytomorphology 27, 187-190 (1977)

17. PALMER, G. H.: Morphology of starch granules in cereal grains and malts. $J$. Inst. Brew. 78 , 326-332 (1972)

18. Pomeranz, Y: Determining the structure of the barley kernel by scanning electron microscopy. Cereal Chem. 49, 1-4 (1972)

19. Pomeranz, Y: Scanning electron microscopy of the endosperm of malted barley. Cereal Chem. 49, 5-19 (1972) 
N. H. Gram: The ultrastructure of germinating barley seeds. II.

20. SAndstedt, R. M: Photomicrographic studies of wheat starch. I. Development of the starch granules. Cereal Chem. 23, 337-359 (1946)

21. Simmonds, D. H.: The ultrastructure of the mature wheat endosperm. Cereal Chem. 49. 212-222 (1972)

22. Williams, J. M. \& C. M. Duffus: Separation and some properties of large and small amyloplasts throughout development in barley endosperm. Plant Physiol. 59, 189-192 (1977)

23. Williams, J. M. \& C. M. Duffus: The development of endosperm amyloplasts during grain maturation in barley. J. Inst. Brew. 84, $47-50(1977)$ 